\title{
Elasto-capillary thinning and breakup of model elastic liquids
}

\author{
Shelley L. Anna \\ Division of Engineering and Applied Sciences, Harvard University, Cambridge, \\ Massachusetts 02138 \\ Gareth H. McKinley ${ }^{\text {a) }}$ \\ Department of Mechanical Engineering, Massachusetts Institute of Technology, \\ Cambridge, Massachusetts 02139
}

(Received 14 June 2000; final revision received 9 October 2000)

\section{Synopsis}

We study the elasto-capillary self-thinning and ultimate breakup of three polystyrene-based ideal elastic fluids by measuring the evolution in the filament diameter as slender viscoelastic threads neck and eventually break. We examine the dependence of the transient diameter profile and the time to breakup on the molecular weight, and compare the observations with simple theories for breakup of slender viscoelastic filaments. The evolution of the transient diameter profile predicted by a multimode FENE-P model quantitatively matches the data provided the initial stresses in the filament are taken into account. Finally, we show how the transient uniaxial extensional viscosity of a dilute polymer solution can be estimated from the evolution in the diameter of the necking filament. The resulting "apparent extensional viscosity" profiles are compared with similar results obtained from a filament stretching rheometer. Both transient profiles approach the same value for the steady state extensional viscosity, which increases with molecular weight in agreement with the Rouse-Zimm theory. The apparent discrepancy in the growth rate of the two transient curves can be quantitatively explained by examining the effective stretch rate in each configuration. Filament thinning studies and filament stretching experiments thus form complementary experiments that lead to consistent measures of the transient extensional viscosity of a given test fluid. (C) 2001 The Society of Rheology. [DOI: 10.1122/1.1332389]

\section{INTRODUCTION}

Small quantities of polymeric additives often have very pronounced effects on fluid motion, and one particular type of flow that has been studied extensively in this regard is the necking and breakup of polymeric liquid jets. Newtonian jets rapidly neck down under capillary action and pinch off into evenly spaced droplets and interspersed satellite droplets due to the well-known Rayleigh-Tomotika instability [Rayleigh (1878); Rayleigh (1892); Tomotika (1935)], whereas even very dilute polymer solutions form stable jets or "beads-on-a-string" structure that persist for a relatively long time [Goldin et al. (1969)]. Extensive reviews of the early literature in the subject are given by Bogy (1979) and in the monograph by Yarin (1993). A comprehensive review of more recent literature on breakup phenomena in Newtonian fluid filaments and jets, including experimental

\footnotetext{
a) Author to whom correspondence should be addressed; electronic mail: gareth@mit.edu
} 
results, numerical simulations, stability analyses, and similarity solutions for the approach to breakup, is provided by Eggers (1997). Early linear stability analyses of viscoelastic jets indicated that they were actually less stable than equivalent Newtonian jets [Middleman (1965); Goldin et al. (1969); Goren and Gottlieb (1982)]. However, Goldin and co-workers noted that nonlinear phenomena become increasingly important as the filament necks down, so that a linear analysis is no longer valid [Goldin et al. (1969)]. Goren and Gottlieb also observed that allowing for a finite initial tension in the filament increased the stability of the filament [Goren and Gottlieb (1982)]. By using a finite element simulation to incorporate nonlinear effects, Bousfield and co-workers were able to simulate the entire transient evolution of a viscoelastic jet as it formed the beads-ona-string configuration [Bousfield et al. (1986)]. More recently, Chang and co-workers have presented an asymptotic analysis and numerical simulations of the elasto-capillary necking dynamics of viscoelastic jets described by the Oldroyd-B model [Chang et al. (1999)].

These stability analyses have shown that the extensional rheological response of a fluid dictates whether or not it will form stable jets and filaments. Building on early work by Chang and Lodge (1972), Ide and White incorporated effects of surface tension and nonlinear extensional rheology to predict the "spinnability" of viscoelastic fluids in imposed uniaxial elongation [Ide and White (1976)]. Renardy $(1994,1995)$ has presented nonlinear analyses of the asymptotic approach to capillary-driven breakup of slender Newtonian and viscoelastic jets using a Lagrangian formulation, and showed that viscoelastic constitutive models predicting unbounded stress growth during extension lead to the formation of filaments that never break while other models with a finite steady state uniaxial extensional viscosity will break in finite time. During the necking and breakup of viscoelastic fluid filaments, elastic tensile stresses resist the pinching caused by capillary action. As a result, tensile stresses in the viscoelastic fluid grow and polymer chains are elongated in the local region near breakup. Since the local flow field in this region is primarily extensional, analysis of necking phenomena provides a plausible way of measuring the extensional response of viscoelastic fluids. Schümmer and Tebel (1983) described a "free jet elongational rheometer," in which the diameter of a periodically forced fluid jet undergoing capillary thinning is monitored as a function of time. Another filament breakup device, referred to as the "microfilament rheometer" was introduced in 1990 [Bazilevsky et al. (1990)], and is similar in operation to the filament stretching device introduced at about the same time [Matta and Tytus (1990); Sridhar et al. (1991); Tirtaatmadja and Sridhar (1993)]. The principal difference between the filament stretching rheometer and the filament breakup rheometer is that in filament stretching devices, a cylindrical liquid bridge is formed between two rigid endplates, which are then actively stretched apart with an exponentially increasing separation profile. In the filament breakup device, these endplates are rapidly separated and then held at a fixed axial separation, and the subsequent evolution of the midfilament diameter is monitored during the process of necking and breakup.

Extensional rheometry utilizing these two devices has progressed in parallel since the early 1990s. Several groups have developed filament stretching rheometers based on the original design of Sridhar and co-workers, and a significant amount of work has been performed to quantify the extensional rheology of dilute polymer solutions as well as to define appropriate experimental technique and interpretation of the resulting measurements [Berg et al. (1994); Solomon and Muller (1996); Spiegelberg et al. (1996); Spiegelberg and McKinley (1996); van Nieuwkoop and Muller von Czernicki (1996); Kolte et al. (1997); Szabo (1997); Anna et al. (1999); Orr and Sridhar (1999); Verhoef et al. (1999)]. Most recently, an interlaboratory comparison of filament stretching data 
for three common test fluids was presented, in which the authors demonstrated that agreement between different designs of filament stretching rheometers is excellent [Anna et al. (2000)].

Using the filament breakup device, Bazilevsky and co-workers $(1990,1997)$ have presented measurements for various polyacrylamide and polyethylene oxide-based polymer solutions, and compared the results with simple theories for the approach to breakup of Newtonian and viscoelastic filaments. Liang and Mackley (1994) presented similar selfthinning measurements for polyisobutylene solutions, and used the evolution in the diameter profile to obtain a characteristic relaxation time for these fluids. They found that the relaxation time $\lambda_{R}$ obtained from filament thinning measurements was nearly a factor of 3 larger than the mean relaxation time $\lambda^{-}$obtained from shear rheology measurements. More recently, Stelter and co-workers (2000) presented thinning measurements for polyacrylamide solutions, and used the transient diameter profiles to compute a relaxation time and an apparent elongational viscosity for these semidilute solutions. Entov and Hinch (1997) used a multimode FENE model to predict the breakup profile of very slender viscoelastic fluid filaments. The authors analyzed the asymptotic behavior in three different regimes of the elasto-capillary flow and predicted the time dependence of the midfilament diameter in each regime. In a recent experimental and numerical study, Kolte and Szabo (1999) found that quantitative agreement with experimental data could not be achieved using this theory unless axial variations in the filament profile and radial variations in the tensile stress difference were also incorporated. Finally, McKinley and Tripathi (2000) also used a filament breakup device to compare measurements with viscous Newtonian fluids to the appropriate similarity solution for the approach to breakup and showed that, for Newtonian filaments, quantitative agreement with the onedimensional theory of Papageorgiou (1995) can be achieved by correctly accounting for the self-similar configuration of the necking filament.

Having demonstrated that measurements of capillary breakup in Newtonian fluids can be successfully described by a one-dimensional theory, we now seek to investigate if the appropriate one-dimensional analysis [Entov and Hinch (1997)] can be used to extract quantitatively accurate values of material parameters for viscoelastic liquids. The analysis of Entov and Hinch for capillary thinning and breakup of elastic filaments is based on a multimode formulation of the FENE-P dumbbell theory, which accurately describes the rheology of infinitely dilute monodisperse homopolymer solutions. Unfortunately, almost all experimental analyses of elasto-capillary thinning to date have used semidilute or concentrated solutions of polydisperse macromolecules. The shear rheology of such materials is typically poorly described by idealized models such as the FENE constitutive equation unless unrealistically low values of the relevant model parameters are chosen [Entov and Hinch (1997)]. It is thus not possible to ascertain whether material properties extracted from measurements of elasto-capillary thinning are consistent with the values of molecular parameters on which the original model and analysis were based. The goal of the present study is thus to investigate the elasto-capillary drainage and breakup of dilute monodisperse polymer solutions which have already been well characterized in viscometric flows using conventional shear rheometry and also in transient uniaxial extension using filament stretching rheometry.

Although the flow in a filament stretching rheometer and in a capillary breakup rheometer are slightly different, both devices generate a uniaxial extensional flow. In the filament stretching device, the characteristic time scale of the flow is well defined, $t_{\text {flow }}=\left(\dot{\varepsilon}_{0}\right)^{-1}$, and thus the flow kinematics and fluid response are relatively straightforward to interpret. However, an ideal filament stretching experiment is practically dif- 


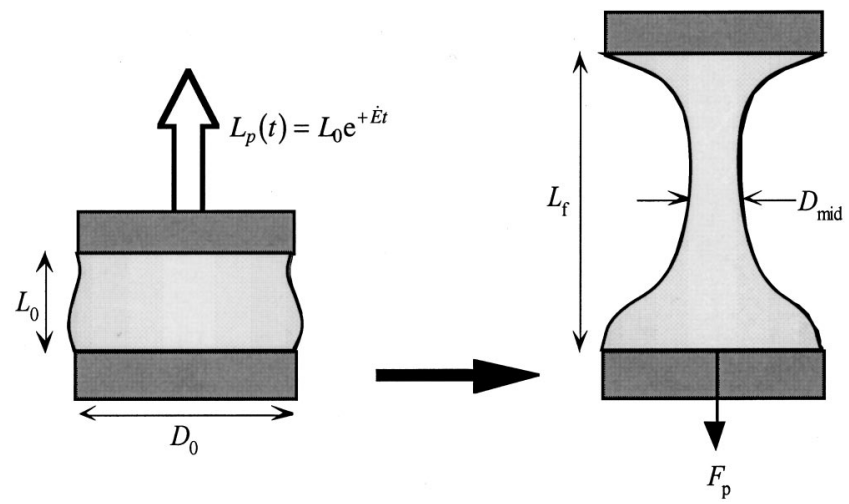

FIG. 1. Schematic diagram of a capillary thinning experiment.

ficult to implement, and several limitations arise from mechanical constraints as well as gravitational sagging effects and elastic instabilities [Anna et al. (2000)]. By contrast, a filament breakup experiment is relatively simple to perform; however, since the fluid column is allowed to spatially rearrange and select its own time scales, the dynamics of the fluid response can be quite complicated and the kinematics are typically time dependent. Analysis and interpretation of filament thinning data is therefore not as straightforward as that of filament stretching data. However, the response of a given fluid to these two different flows depends only on its microstructure as encoded in the constitutive equation, and thus we expect a correct analysis of each experiment to yield consistent rheological information for a given fluid.

In this study, we examine the filament stretching and breakup responses of three different polystyrene-based Boger fluids, in which the polymer concentration is fixed at $0.05 \mathrm{wt} \%$ and the polymer molecular weights are $2.0,6.5$, and 20 million $\mathrm{g} / \mathrm{mol}$. These three Boger fluids are the same well-characterized test fluids used in the interlaboratory comparison of filament stretching devices mentioned above [Anna et al. (2000)]. In the following section, we present measurements of the evolution in the midfilament diameter profiles $D_{\text {mid }}(t)$ observed during elasto-capillary thinning experiments for the three different Boger fluids. We compare the characteristic relaxation time obtained from this data to the relaxation times obtained from conventional viscometric experiments. In Sec. III, we compare these experimental observations to theoretical predictions using a multimode FENE model, and in Sec. IV, we show how the force balance on the filament can be used to reexpress midfilament diameter profiles in the form of a transient extensional viscosity. Finally, we compare the molecular weight dependence of the steady-state extensional viscosity obtained from the asymptotic behavior of filament breakup experiments to the behavior predicted from kinetic theory for bead-spring chains.

\section{FILAMENT THINNING AND BREAKUP EXPERIMENTS}

\section{A. Experiment setup}

Although several devices have been developed to specifically make measurements of visco-elasto-capillary thinning [Bazilevsky et al. (1990); Kolte and Szabo (1999); McKinley and Tripathi (2000); Stelter et al. (2000)], the filament breakup experiments performed in the present study were actually carried out in a filament stretching apparatus that has been described in detail elsewhere [Anna et al. (1999, 2000)]. A schematic diagram of the experimental configuration is shown in Fig. 1. A nearly cylindrical sample 


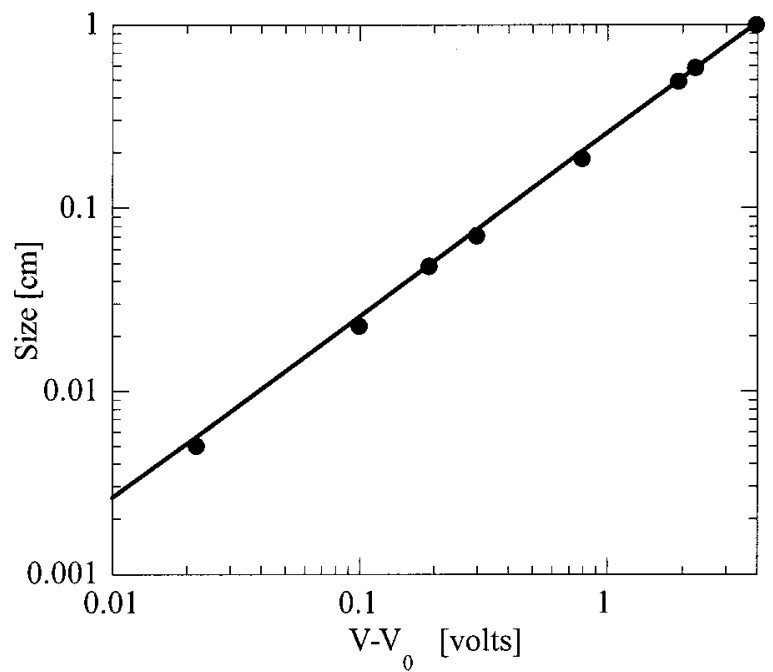

FIG. 2. Calibration of diameter sensor using optical fibers of known size. The measured voltage $(\mathbf{\bullet})$ increases linearly as diameter increases, as shown by the solid fitted line.

of fluid initially fills the gap between two rigid, circular endplates with diameter $D_{0}$ and initial separation $L_{0}$. The endplates are then moved apart to a final separation $L_{f}$ with an exponentially increasing separation profile, $L_{p}(t)=L_{0} \exp (+\dot{E} t)$, where $\dot{E}$ is the stretch rate. During the short period of stretching, both the midfilament diameter $D_{\text {mid }}$ and the tensile force at the endplate $F_{p}$ are monitored but once the endplates have stopped moving, the force rapidly decays to zero, and the midfilament diameter alone is monitored until the fluid column breaks. The filament stretching apparatus was used so that the rate of separation of the endplates could be precisely controlled, and so that the initial stresses in the filament could be monitored. However, we have also performed analogous filament breakup experiments on the same test fluids in a microfilament rheometer designed by Entov and co-workers [Bazilevsky et al. (1990)] in which the rate of axial separation is not precisely controlled, and the transient midfilament diameter profiles following cessation of stretching agreed quantitatively between the two devices. The initial axial separation of the plates may thus be viewed as the elongational equivalent of a "step-strain" experiment, provided that the separation rate $\dot{E}$ is much faster than the viscoelastic relaxation time in the fluid $\left(\dot{E} \gg 1 / \lambda_{1}\right)$ and the timescale for capillary drainage $\left(\dot{E} \gg 2 \sigma / \eta_{0} D_{0}\right)$.

The diameter sensor used in these experiments is a laser micrometer which computes the size of an object in its path based on the intensity of light entering the sensing element (Omron Z4LA). This sensor is calibrated periodically using transparent optical fibers that mimic the opacity of the fluid filaments. The calibrated resolution of the sensor is approximately $20 \mu \mathrm{m}$, and a representative calibration plot for the sensor is shown in Fig. 2. The Hencky strain $\varepsilon(t)$ experienced by the fluid element at the axial midplane at time $t$ can be defined using the midfilament diameter, in the same way as in a filament stretching experiment, by

$$
\varepsilon=2 \ln \left(D_{0} / D_{\text {mid }}(t)\right)
$$


TABLE I. Viscometric properties of SM Boger fluids. The density of each fluid is $\rho=1026 \mathrm{~kg} / \mathrm{m}^{3}$, the surface tension is $\sigma=0.0378 \mathrm{~N} / \mathrm{m}$, and the viscosity of the solvent is $\eta_{s}=34.0 \mathrm{~Pa} \mathrm{~s}$.

\begin{tabular}{ccccccc}
\hline \hline & $M_{w}(\mathrm{~g} / \mathrm{mol})$ & $\eta_{0}(\mathrm{~Pa} \mathrm{~s})$ & $\lambda_{Z}(\mathrm{~s})$ & $L$ & $t_{\text {cap }}(\mathrm{s})$ & Bo \\
\hline SM-1 & $2.0 \times 10^{6}$ & 39.2 & 3.7 & 88 & 1.56 & 0.60 \\
SM-2 & $6.5 \times 10^{6}$ & 46.1 & 31.1 & 164 & 1.83 & 0.60 \\
SM-3 & $2.0 \times 10^{7}$ & 55.5 & 155 & 277 & 2.20 & 0.60 \\
PS oil & - & 32.5 & - & - & 3.01 & 3.26 \\
\hline \hline
\end{tabular}

The maximum measurable Hencky strain in the filament thinning experiments can be estimated using the value of the midfilament diameter immediately after stretching has stopped and the minimum diameter measurable by the sensor. This maximum Hencky strain is given by

$$
\varepsilon_{\max } \approx 2 \ln \left(\frac{1.5 \mathrm{~mm}}{20 \mu \mathrm{m}}\right)=8.6
$$

Note that this estimate is based on the calibrated minimum diameter of $20 \mu \mathrm{m}$, but that voltage values below the minimum calibration point are measured during a filament breakup experiment. Since the calibration curve shown in Fig. 2 appears to be linear even for low voltages, reliable diameter values as low as $5 \mu \mathrm{m}$ are obtained in practice, corresponding to Hencky strains as large as $\varepsilon_{\max } \approx 11-12$. Large fluid strains can thus be attained without the need for extremely large filament stretching instrumentation.

The test fluids used in this study are the same materials employed in the accompanying interlaboratory comparison of filament stretching devices [Anna et al. (2000)]. The three fluids, denoted SM-1, SM-2, and SM-3, are polystyrene-based Boger fluids consisting of high-molecular weight polystyrene dissolved in a Newtonian styrene oil. The composition, equilibrium properties, and shear rheology of these three fluids has been characterized thoroughly and is described in detail in the interlaboratory comparison paper of Anna et al. (2000). The molecular weights of the polystyrene solutes are 2.0, 6.5 , and 20 million $\mathrm{g} / \mathrm{mol}$, and the concentration of polystyrene is fixed for all three fluids at $0.05 \mathrm{wt} \%$, corresponding to dimensionless concentrations of $c / c^{*}=0.44,0.87$, and 1.50 , respectively. Under equilibrium conditions these elastic fluids thus span the dilute and semidilute range. The linear viscoelastic behavior of all three fluids was found to be well described by dilute solution theory with a Zimm spectrum of relaxation times, with an additional contribution from solvent elasticity that becomes important only at high deformation rates. The relevant fluid properties for the present study are reproduced in Table I, including zero-shear-rate viscosity $\eta_{0}$, longest relaxation time $\lambda_{Z}$, and finite extensibility parameter $L$. In addition, the density $\rho$, surface tension $\sigma$, and solvent viscosity $\eta_{s}$ are given. The surface tension of the fluids was measured using a Wilhelmy plate in a Krüss K-10 tensiometer, and was found to be identical for all three Boger fluids, and for the Newtonian solvent. This is not surprising since the chemical composition of the solute and solvent is identical.

In addition to the relaxation time of the fluid, the other characteristic time scale of importance in elasto-capillary thinning studies is the capillary time scale. This capillary time relates the relative effects of capillary and viscous forces for a given fluid and is given by 
TABLE II. Experimental parameters for filament thinning experiments. The test conditions for the four fluids were the same, with a constant stretch rate $\dot{E}=1.0 \mathrm{~s}^{-1}$, an initial aspect ratio $\Lambda_{0}=1.0$, and a final aspect ratio $\Lambda_{f}=2.72$.

\begin{tabular}{ccccc}
\hline \hline & $D_{0}(\mathrm{~mm})$ & $D_{1}(\mathrm{~mm})$ & $A_{z z}^{0}$ & $\mathrm{~T}\left({ }^{\circ} \mathrm{C}\right)\left(a_{T}\right)$ \\
\hline SM-1 & 3.0 & 1.564 & 47.4 & $20.9(2.64)$ \\
SM-2 & 3.0 & 1.364 & 221 & $21.0(2.55)$ \\
SM-3 & 3.0 & 1.320 & 712 & $21.1(2.51)$ \\
PS oil & 7.0 & 3.042 & - & $25.0(1.00)$ \\
\hline \hline
\end{tabular}

$$
t_{\text {cap }}=\frac{\eta_{0}}{\left(2 \sigma / D_{0}\right)} .
$$

Corresponding values for each fluid are given in Table I. For dilute polymer solutions described by the Zimm model, increasing the molecular weight of the polymer (i.e., solute) does not increase the total solution viscosity significantly since $\left(\eta_{0}-\eta_{s}\right)$ $\propto M_{w}^{1 / 2}$, and the capillary time is roughly equal for all three test fluids. By contrast, the relaxation time increases by more than a factor of 40 for an order of magnitude change in molecular weight, since $\lambda_{Z} \propto M_{w}^{3 / 2}$.

Although analysis of filament thinning experiments assumes axial symmetry about the midplane, gravitational effects break this symmetry and lead to a weak axial flow; the filament "sags", such that more than half the initial volume of fluid is found below the midplane. The Bond number

$$
\mathrm{Bo}=\frac{\rho g D_{0}^{2}}{4 \sigma},
$$

provides an estimate of the relative magnitude of this sagging effect compared with the opposing capillary forces. The Bond number for each Boger fluid is identical, and is given in Table I. The breakup experiments with Newtonian fluids were performed using $7.0 \mathrm{~mm}$ plates, corresponding to $\mathrm{Bo} \approx 3.6$, and some axial asymmetry was readily observable before the breakup event. The extent of this axial drainage depends not only on the magnitude of the Bond number but also on the total time the liquid bridge remains connected. Addition of small amounts of high-molecular-weight polymer increases the filament lifetime dramatically and accordingly the axial asymmetry was also found to increase. Similar effects were observed in simulations by Kolte and Szabo (1999). To minimize this gravitational sagging, experiments with the SM fluids were performed using $3.0 \mathrm{~mm}$ plates resulting in a reduction of the Bond number to $\mathrm{Bo} \approx 0.60$.

Test parameters for all three Boger fluids and the Newtonian solvent are given in Table II. For each fluid, the endplate diameter $D_{0}$ is given along with the value of the midfilament diameter $D_{1}$ measured optically at the time stretching stops. For a rapid step-strain, the initial deformation of the fluid column should be well described by the "reverse squeeze flow" solution described by Spiegelberg and McKinley (1996). Ignoring the effects of gravity, the midpoint filament diameter at the instant stretching stops should thus be given by $D_{\text {mid }}\left(t_{1}\right) \approx D_{0}\left(\Lambda_{0} / \Lambda_{f}\right)^{4 / 3}=1.42 \mathrm{~mm}$. This is in good agreement ( $\pm 10 \%$ ) with the values of $D_{1}$ observed experimentally.

The temperature $T$ of each experiment is also given in Table II, along with the corresponding shift factor $a_{T}$ that is used in time-temperature superposition. The thermal 
(a) Styrene Oligomer

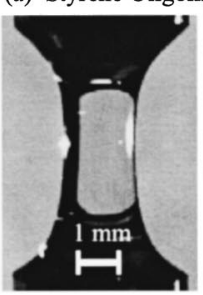

$t=0 \mathrm{~s}$

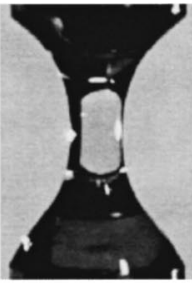

$t=2.1 \mathrm{~s}$

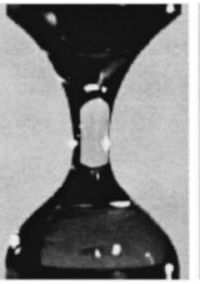

$t=4.2 \mathrm{~s}$

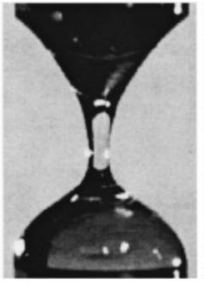

$t=6.3 \mathrm{~s}$

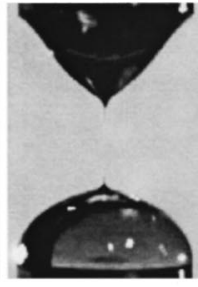

$t=8.6 \mathrm{~s}$

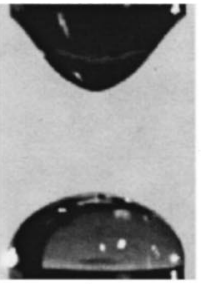

$t=10.5 \mathrm{~s}$

(b) SM-1 Boger Fluid: $0.05 \mathrm{wt} . \% 2 \times 10^{6} \mathrm{~g} / \mathrm{mol}$ polystyrene in styrene oligomer

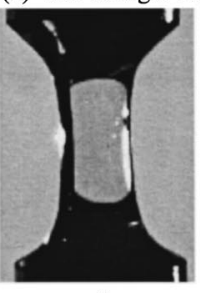

$t=0 \mathrm{~s}$

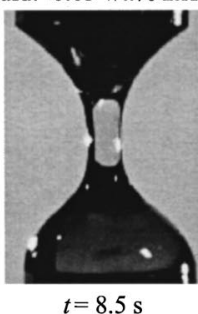

$t=8.5 \mathrm{~s}$

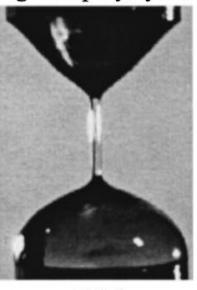

$t=17.0 \mathrm{~s}$

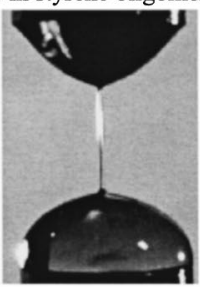

$t=25.5 \mathrm{~s}$

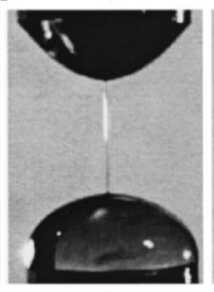

$t=34.0 \mathrm{~s}$

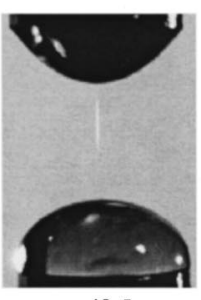

$t=42.5 \mathrm{~s}$

FIG. 3. Sequence of video images showing capillary thinning and breakup for Newtonian and viscoelastic filaments. The plate diameters in both sequences is $D_{0}=3 \mathrm{~mm}$. While Newtonian filaments (top row) are always axially nonuniform, Boger fluids (bottom row) form nearly cylindrical filaments that persist much longer than a Newtonian filament with the same viscosity.

sensitivity of these fluids is determined through viscometric measurements, and is described in detail elsewhere [Anna et al. (2000)]. Time-temperature superposition is used in the filament necking experiments to shift the experimental elapsed times to those corresponding to a reference temperature $T_{0}$ according to the relationship

$$
t\left(T_{0}\right)=\frac{t(T)}{a_{T}} .
$$

Finally, the stretching parameters imposed for all four necking experiments were equivalent, with equal stretching rates $\dot{E}$ and equal initial and final aspect ratios, which we denote $\Lambda_{0}$ and $\Lambda_{f}$, respectively.

\section{B. Experimental results}

Newtonian and viscoelastic fluid filaments neck and break in a qualitatively different manner. Both the axial diameter profile and the time evolution in the midfilament diameter are significantly different for each type of fluid. Figure 3 shows a time sequence of filament breakup images taken with a charge coupled device camera for: (a) the Newtonian styrene oil used in this study and (b) the SM-1 polymer solution. The axial profile of the Newtonian filament is always inhomogeneous, and the minimum diameter is always close to the midplane (although gravitational effects cause the actual minimum to be slightly above the midplane). The axial profile of the viscoelastic filament is initially similar to the Newtonian profile, but rapidly evolves into an axially uniform cylindrical filament that connects the two roughly hemispherical fluid reservoirs near the endplates. The time to breakup for the elastic Boger fluid is significantly longer than that of a Newtonian fluid, even though the viscosities and capillary times are approximately equal. 
Several authors have presented simple theories and similarity solutions that predict the transient midfilament diameter for both Newtonian and viscoelastic filaments [Bazilevsky et al. (1990); Renardy (1994, 1995); Brenner et al. (1996); Bazilevsky et al. (1997); Eggers (1997); Entov and Hinch (1997)]. The solutions for breakup of a Newtonian filament all predict a linearly decreasing diameter, with a form given by

$$
D_{\text {mid }}(t)=\alpha\left(\frac{2 \sigma}{\eta_{s}}\right)\left(t_{b}-t\right),
$$

where $t_{b}$ is the time to breakup; however, the value of the numerical prefactor $\alpha$ is different for each solution. McKinley and Tripathi (2000) have recently shown that the similarity solution to the Stokes equations given by Papageorgiou (1995), with $\alpha=0.0709$, agrees well with experiments using viscous Newtonian liquids.

By contrast to Newtonian fluids, the midfilament diameter of an Oldroyd-B fluid filament is predicted to decrease exponentially with time [Bousfield et al. (1986); Bazilevsky et al. (1990); Renardy (1994); Bazilevsky et al. (1997); Entov and Hinch (1997)]. If viscous stresses are assumed to be negligible, and elastic and capillary contributions to the total force balance each other, then the mid-filament diameter is predicted to decrease according to the form,

$$
D_{\text {mid }}(t)=\left(\frac{\eta_{p} D_{1}^{4}}{2 \bar{\lambda} \sigma}\right)^{1 / 3} \exp \left(-\frac{t}{3 \bar{\lambda}^{-}}\right),
$$

where $\eta_{p}=\eta_{0}-\eta_{s}$ is the polymeric contribution to the viscosity, $\bar{\lambda}$ is the characteristic relaxation time of the Oldroyd filament, and $D_{1}$ is the midpoint diameter following cessation of stretching. The elastic modulus of the material is given by $G \equiv \eta_{p} / \bar{\lambda}$ and when written in dimensionless form the prefactor in Eq. (7) may be viewed as an elastocapillary number $G D_{1} / 2 \sigma$. The analysis above applies to elastic fluids described by the single time constant of the Oldroyd-B model. However, in reality, dilute polymer solutions have a spectrum of time constants and it remains to determine which moment of this spectrum is actually measured in capillary-thinning experiments. For a dilute suspension of noninteracting FENE dumbbells with a distribution of moduli $G_{i}$ and time constants $\lambda_{i}$, Entov and Hinch (1997) show that for "intermediate elastic times" (i.e., after viscous effects have become negligible and before finite extensibility of the dumbbells becomes important), Eq. (7) can be generalized to

$$
\frac{D_{\text {mid }}(t)}{D_{1}}=\left(\sum_{i}\left(\frac{G_{i} D_{1}}{2 \sigma}\right) \exp \left(-t / \lambda_{i}\right)\right)^{1 / 3} .
$$

Precisely the same equation can be obtained from the Rouse-Zimm model for an $\mathrm{N}$-beadspring chain except that $G_{i}$ and $\lambda_{i}$ for each mode are now related to each other by relationships of the form

$$
\begin{gathered}
\lambda_{i}=\lambda_{Z} / i^{2+\tilde{\sigma}}, \\
G_{i}=n k_{B} T,
\end{gathered}
$$

where $\tilde{\sigma}$ is a measure of the hydrodynamic interaction. The dilute polymer solutions studied in the present work are well described by the Zimm model with $\tilde{\sigma} \approx-0.4$. As a result of the broad separation of timescales in the Zimm spectrum, Eq. (8) can be simplified at "long" times since the contribution of the higher modes rapidly decays. For 


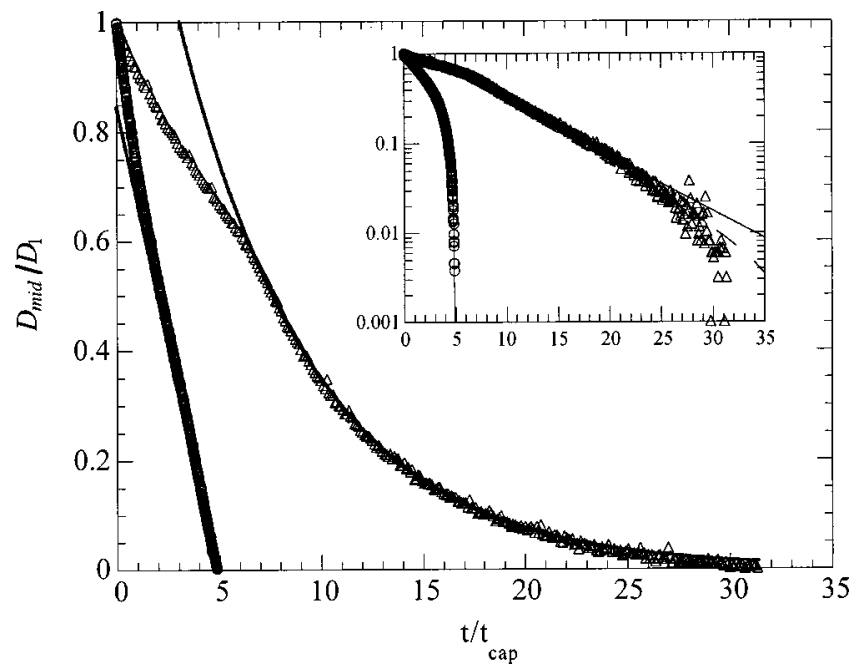

FIG. 4. Transient midfilament diameter profiles for thinning filaments of Newtonian styrene oligomer $(O)$ and SM-1 Boger fluid $(\triangle)$. Newtonian filaments break nearly linearly with time, while Boger fluid filaments break nearly exponentially with time. Solid curves show linear and exponential fits to the respective measured data. The inset shows the same data on a semilogarithmic scale, and the dashed line corresponds to a linear fit to the data obtained for the SM-1 fluid at long times $t / t_{\text {cap }} \geqslant 20$.

$t>\lambda_{Z}$ the column necks sufficiently slowly that all of the elastic stress is carried by the longest mode $(i=1)$ and the additional modes augment the (now negligible) viscous response of the solvent. Equation (8) then simplifies again to the form of Eq. (7) with $\bar{\lambda}$ replaced by $\lambda_{Z}$. Regression of experimental data for dilute polymer solutions undergoing elasto-capillary thinning to Eq. (7) should thus yield estimates of the Zimm (longest) time constant.

Transient midfilament diameters measured for the Newtonian styrene oil and the SM-1 Boger fluid are shown in Fig. 4. As predicted from Eqs. (6) and (7), the midpoint diameter profile of the Newtonian filament decreases approximately linearly with time, while measurements of the filament evolution for the Boger fluid decay approximately exponentially with time. The inset to Fig. 4 shows that the diameter deviates from the exponential form of Eq. (7) at long times. Entov and Hinch (1997) show that this deviation arises from the finite extensibility of the molecules and the bounded nature of the associated elastic extensional stress. Asymptotic analysis for both the FENE-P and Giesekus models [Renardy (1995); Entov and Hinch (1997)] show that in this regime the radius is expected to once again decrease linearly with time until the filament breaks. The dashed line shown in Fig. 4 corresponds to a linear fit to the data for $t / t_{\text {cap }} \geqslant 20$ from which we can extract the critical time for breakup of the filament. We return to this ultimate failure event in Sec. IV.

The values of $\alpha$ and $\lambda^{-}$in Eqs. (6) and (7) are each determined from a linear regression to the experimental data shown, and these values are reported in Table III along with values of the elasto-capillary number $\left(\eta_{p} D_{1} / 2 \sigma \bar{\lambda}\right)$ at the moment the initial step-strain motion ceases. The value of $\alpha$ for the Newtonian styrene oil agrees with the Papageorgiou prediction to within experimental error, in agreement with the results reported by McKinley and Tripathi. In addition, the characteristic relaxation time $\bar{\lambda}$ for filament thinning of the SM-1 fluid agrees with the longest relaxation time $\lambda_{Z}$, obtained from shear rheology, to within experimental error. In Fig. 5 we present the measured midfila- 
TABLE III. Measured and computed properties of SM Boger fluids as determined from capillary-thinning experiments.

\begin{tabular}{ccccccccc}
\hline \hline & & \multicolumn{3}{c}{ Experiments } & & \multicolumn{3}{c}{ Simulations } \\
\cline { 3 - 4 } Fluid & $\frac{\eta_{p} D_{1}}{2 \bar{\lambda} \sigma}$ & $\bar{\lambda}(\mathrm{s})$ & $S \equiv \frac{t_{b}}{\lambda_{Z}}$ & $\operatorname{Tr}_{\infty}$ & & $\bar{\lambda}(\mathrm{s})$ & $S \equiv \frac{t_{b}}{\lambda_{Z}}$ & $\operatorname{Tr}_{\infty}$ \\
\hline SM-1 & 0.0337 & 3.19 & 15.8 & 1110 & & 3.58 & 14.6 & 1200 \\
SM-2 & 0.0079 & 27.8 & 16.0 & 8010 & & 30.9 & 15.3 & 8850 \\
SM-3 & 0.0025 & 152 & 17.9 & 41100 & & 152 & 15.2 & 33300 \\
PS oil & \multicolumn{3}{c}{$\alpha=0.075 \pm 0.013$} & & $\alpha=0.0709$ & & \\
\hline \hline
\end{tabular}

ment diameter results for all three SM fluids. All three profiles are observed to decay approximately exponentially over almost 2 orders of magnitude in $D_{\text {mid }}(t)$. Regression to the measured data for the SM-2 and SM-3 fluids yielded values of $\lambda^{-}$that agree with the values of $\lambda_{Z}$ obtained from shear rheology measurements.

As the molecular weight of the polymeric solute increases, the viscosity and relaxation time of the test fluids also increases. For viscous Newtonian fluids the appropriate timescale for characterizing capillary breakup is the capillary time $t_{\text {cap }}=\eta_{0} D_{0} / 2 \sigma$ given in Table I. However, it is clear from Figs. 4 and 5 that this is not the appropriate scale for elasto-capillary thinning since from the Zimm theory it exhibits a very weak dependence on molecular weight $\left(\eta_{0}-\eta_{s}\right) \sim M_{w}^{3 \nu-1}$, where $\nu$ is the excluded volume parameter. Analysis of Eqs. (7) and (8) suggests that for dilute polymer solutions a more appropriate scale is the longest (Zimm) time constant which exhibits a more pronounced dependence on chain length $\left(\lambda_{Z} \sim M_{w}^{3 \nu}\right)$. The same midfilament diameter data that appears in Fig. 5 is replotted in Fig. 6 with the time axis rescaled with the longest relaxation time of the fluid. In this case, the three necking profiles nearly collapse onto a "master curve." Finally, we note that the ratio of the time to breakup and the longest relaxation time, defined previously by Bazilevsky and co-workers (1997) as the parameter $S \equiv t_{b} / \lambda_{Z}$,

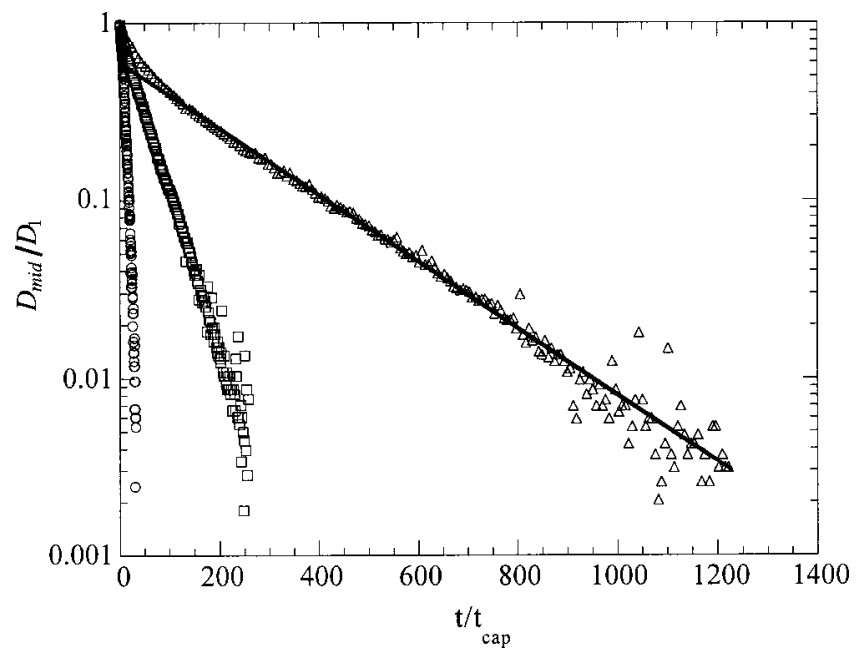

FIG. 5. Transient midfilament diameter profiles for breaking filaments of SM Boger fluids. Breakup time increases dramatically as the polymer molecular weight increases [SM-1 (O); SM-2 ( $\square)$; SM-3 $(\triangle)$ ]. 


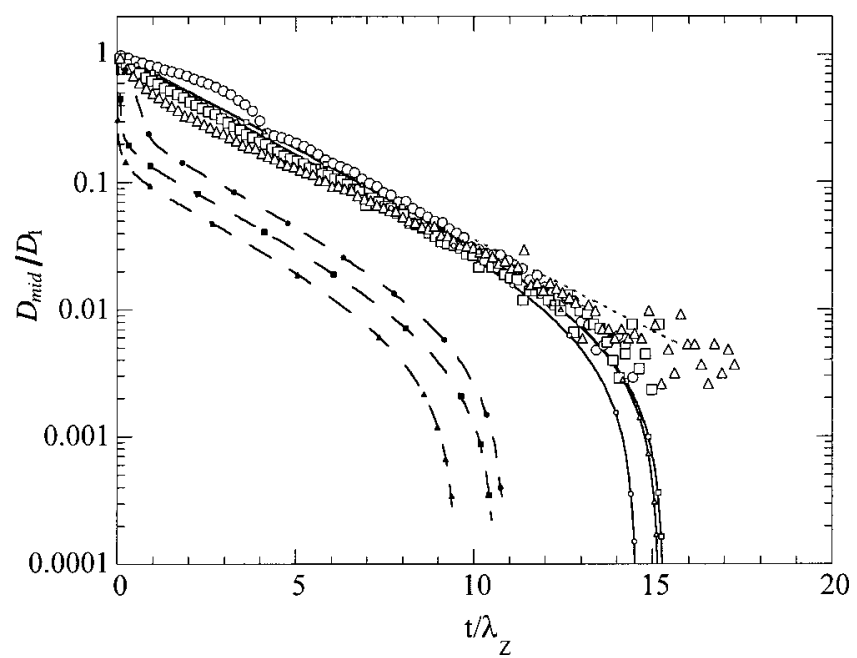

FIG. 6. Comparison of measured midfilament diameter profiles for elastic fluid filaments with predictions from a multimode FENE-P model [SM-1 $(\bigcirc)$; SM-2 $(\square)$; SM-3 $(\triangle)$ ]. Two sets of numerical simulations are shown by the lines (with small symbols corresponding to material parameters for SM-1, -2 , and -3 , respectively). Broken lines correspond to zero initial stresses in the filament; solid lines correspond to an initial deformation given by Eq. (18) in the text.

ranges between 15 and 18 for the three fluids considered here. This range is consistent with that previously reported for other dilute polymer solutions [Bazilevsky et al. (1997)] and is noticeably longer than the values for semidilute or concentrated solutions in which finite extensibility effects become pronounced much earlier.

We have also explored the effect of the total initial stretch $\Lambda_{f} \equiv \Lambda_{0} \exp \left(\varepsilon_{f}\right)$ imposed on the filament. For large initial axial strains $\varepsilon_{f} \geqslant 2$, filament stretching experiments show that significant strain hardening in the elastic stress is to be expected. The slow relaxation of this initial stress delays the necking of the filament. Conversely, for small strains $\varepsilon_{f} \leqslant 1$, the small amplitude of the initial perturbation in the shape of the liquid bridge means that gravitational "slumping" and the quasistatic fluid reservoirs at each endplate exert considerable influence on the necking dynamics. The evolution of the filament is thus poorly described by the uniform axial thread model. Our experiments suggest that the optimal range of imposed axial Hencky strains is $1 \leqslant \varepsilon_{f} \leqslant 2$. As a consequence of the no-slip boundary condition imposed by the endplates, a cylindrical sample does not deform affinely, and the true strain accumulated by the fluid element at the axial midplane is, in fact, $\varepsilon_{\text {mid }}\left(t_{1}\right) \equiv 2 \ln \left(D_{0} / D_{1}\right) \cong 1.5$ (for our experiments), as given by the reverse squeeze flow solution [Spiegelberg et al. (1996)].

\section{MODELING ELASTO-CAPILLARY THINNING AND BREAKUP}

The expression given in Eq. (7) is valid at intermediate times during the elastocapillary thinning of a Boger fluid filament. In this regime, viscous contributions to the stress are negligible, and the elastic stresses in the fluid exactly balance and resist the "pinching"' action of the capillary pressure. In order to fully describe the entire evolution of the midfilament diameter, it is necessary to incorporate viscous stresses, which play a role in the initial transient regime, as well as the finite extensibility of the polymer chains, which become important at late times, as the filament ruptures in finite time. Entov and Hinch (1997) have already investigated in detail the filament breakup of dilute polymer 
solutions, using a multimode FENE model to examine the consequences of a spectrum of relaxation times and the effects of finite molecular extensibility. Each of the regimes described above were considered in that study, and the asymptotic behavior of the midfilament diameter in each regime was calculated.

In the present study, we use the method described by Entov and Hinch to predict the filament thinning behavior of the SM Boger fluids. We use a multimode FENE-P model, with a spectrum of relaxation times corresponding to the Zimm spectrum obtained from the linear viscoelastic data for each fluid. The evolution equations for the $i$ th mode can be written in terms of the conformation tensor $\mathbf{A}_{i}$, as follows:

$$
\mathbf{A}_{i,(1)}=-\frac{1}{\lambda_{i}}\left(f_{i} \mathbf{A}_{i}-\mathbf{I}\right),
$$

where the subscript (1) denotes the upper-convected derivative [Bird et al. (1987)], and I is the unit tensor. The quantity $f_{i}$ is defined by

$$
f_{i}=\frac{1}{1-\operatorname{tr} \mathbf{A}_{i} / L_{i}^{2}},
$$

where $L_{i}$ is the finite extensibility of the given mode, and is related to the finite extensibility parameter $L$ according to

$$
L_{i}=\frac{L}{i^{\nu}},
$$

where $\nu$ is the excluded volume exponent for the SM Boger fluid, which has been characterized elsewhere [Anna et al. (2000)]. For an ideal uniaxial extensional flow, the polymeric contribution to the tensile stresses can now be computed from the above quantities using the following relationship:

$$
\Delta \tau_{p} \equiv\left[\tau_{z z}-\tau_{r r}\right]=\sum_{i=1}^{N_{m}} n k T f_{i}\left(A_{z z, i}-A_{r r, i}\right),
$$

where $N_{m}$ is the number of relaxation modes, and the elastic modulus of all modes is given by $G \equiv n k T=\left(\eta_{0}-\eta_{s}\right) / \lambda_{Z} \Sigma_{i} 1 / i^{2+\tilde{\sigma}}$. We use independently measured values of the SM fluid properties that appear in the above equations, with no adjustable parameters.

At each timestep, the polymeric tensile stress difference $\Delta \tau_{p}$ is computed, and the corresponding strain rate $\dot{\varepsilon}$ is obtained from an axial force balance on the filament. The force balance includes viscous, elastic, and capillary terms, and ignoring axial curvature effects, is given by

$$
3 \eta_{s} \dot{\varepsilon}=\left[\frac{2 \sigma}{D_{\text {mid }}}-\Delta \tau_{p}\right]
$$

For slender filaments the rate of strain in a fluid element can be expressed in terms of the rate of evolution of the filament diameter by

$$
\dot{\varepsilon}=-2 \frac{d \ln \left(D_{\text {mid }} / D_{0}\right)}{d t} .
$$

This definition can be combined with Eq. (14) to obtain a differential equation describing the transient evolution of the diameter. Equations (10)-(15) form a coupled set of (2 
$N_{m}+1$ ) ordinary differential equations (ODEs) for $D_{\text {mid }}(t), A_{z z, i}(t)$, and $A_{r r, i}(t)$ which can be integrated using standard routines for stiff ODEs.

The results of simulations using $N_{m}=8$ are depicted in the three lower (broken) curves shown in Fig. 6 . The predicted curves have a qualitatively similar shape to the experimental curves, but the time to breakup is significantly underpredicted, and thus the magnitude of the midfilament diameter is also underpredicted at all times. This discrepancy can be attributed to the fact that in the original model of Entov and Hinch the initial viscoelastic stresses in the filament at the cessation of stretching are assumed to be zero. This leads to an initial "viscous regime" in the model during which only the viscous response of the filament resists capillarity and the thread thus rapidly constricts. However, in reality, the necked fluid filament has been formed by an initial stretching process, and viscoelastic stresses will also have built up in the filament. Entov and Hinch (1997) recognized the importance of this initial deformation and used theoretical estimates for the initial conditions when comparing their computations with the experiments of Liang and Mackley (1994).

The capillary thinning and breakup experiments described in the present study were performed in a filament stretching device equipped with a sensitive force transducer in order to allow the evolution in the tensile force in the filament to be monitored during the initial stretching process. The value of the tensile force and the midpoint diameter measured shortly after cessation of stretching (at time $t=t_{1}$ ) can be utilized in order to estimate an initial stress in the filament, which can then be used as an initial condition to predict the subsequent evolution in the midfilament diameter profile. Several assumptions must be made to obtain suitable initial conditions for $2 N_{m}+1$ ODEs from two experimental observables, however, given the hyperbolic nature of the constitutive equations, using any nonzero estimate should improve the predicted transient profiles.

In addition to the above procedure, we have also developed an alternate approach to the analysis of capillary thinning data for dilute polymer solutions which does not require the measurement of the tensile force at the cessation of stretch. The most noticeable deviation between the computed and measured filament thinning profiles shown in Fig. 6 is the absence (in the experiments) of the initial viscous regime at short times during which the numerically computed values of $D_{\text {mid }}(t)$ rapidly decrease. This conspicuous lack of an initial visco-capillary regime is also evident in the experimental measurements of Kolte and Szabo (1999) and Stelter et al. (2000). All experimental measurements of capillary thinning using dilute elastic polymer solutions show that, following the cessation of the rapidly imposed endplate deformation, the midpoint radius of the viscoelastic thread in fact decreases in a smooth manner which is approximately exponential in form.

Entov and Hinch sought analytic solutions in which first the viscous and capillary terms balance each other (since the polymer chains in the fluid column were assumed to initially be undeformed), followed by an elasto-capillary regime in which the viscous term is negligibly small and the polymeric stresses balance the capillary term. However, in a dilute polymer solution with a viscous solvent, all terms in Eq. (14) may be important at the beginning of the self-thinning process. During the short, but finite, time required for the cessation of stretching the liquid bridge formed between the two endplates rearranges so that the contributions to the total force in the column given by Eq. (14) balance each other. We therefore seek a solution to Eqs. (10)-(15) in which all terms are retained and are initially of equal magnitude.

Since the flow is extensional in nature and the fluid column selects a stretching rate that is just sufficient to overcome the entropic relaxation of the longest mode (with time constant $\lambda_{Z}$ ) the polymeric contribution to the stress in Eq. (13) is dominated by the first 
term and all other modes relax so that $A_{z z, i} \approx A_{r r, i}$ for $i \neq 1$. If the deformation rate of the bridge is initially constant, as found in the experiments, then the $z z$ component of Eq. (10) can be combined with Eq. (15) and integrated directly (taking $f_{1} \approx 1$ since $L_{1}$ is large) to give

$$
\frac{A_{z z}(t)}{A_{z z}^{0}}=\left(\frac{D_{1}}{D_{\text {mid }}(t)}\right)^{4} \exp \left(-t / \lambda_{Z}\right)
$$

where $A_{z z}^{0}$ is the initial value of the polymeric stretch at the end of the imposed stretching and beginning of capillary thinning. As first discussed by Entov and Hinch, a selfconsistent balance of the elastic and capillary terms in Eq. (14) following the initial viscously dominated response leads to $A_{z z}(t) \sim \exp \left(t / 3 \lambda_{Z}\right), D_{\operatorname{mid}} \sim \exp \left(-t / 3 \lambda_{Z}\right)$ and hence $\dot{\varepsilon}=2 /\left(3 \lambda_{Z}\right)$.

The experimental data in Fig. 6 show that the rate of necking immediately following the cessation of stretching is indeed approximately constant and the same as the value observed at later times; we thus substitute $\dot{\varepsilon}=2 /\left(3 \lambda_{Z}\right)$ into Eq. (14). Combining with Eq. (16) and rearranging leads to

$$
\frac{2 \eta_{s}}{G \lambda_{Z}} \frac{D_{\text {mid }}(t)}{D_{1}}=\left[\frac{2 \sigma}{G D_{1}}-A_{z z}^{0}\left(\frac{D_{1}}{D_{\text {mid }}(t)}\right)^{3} \exp \left(-t / 3 \lambda_{Z}\right)\right] .
$$

It is clear from Eq. (17) that at early times $\left(t / \lambda_{Z} \ll 1\right)$ when $D_{\text {mid }}(t) / D_{1} \sim O(1)$ the viscous response of the solvent on the left-hand side is not negligible unless $\eta_{S}$ $\ll\left(G \lambda_{Z}\right)$. This is not the case for dilute polymer solutions (although it may be a more reasonable approximation for semidilute or concentrated solutions). During the cessation of stretching, the fluid column will thus rearrange so that all three terms in Eq. (17) balance, corresponding to an initial value of the axial stretch given by

$$
A_{z z}^{0}=\left[2 \sigma /\left(G D_{1}\right)-2 \eta_{s} /\left(G \lambda_{Z}\right)\right]
$$

The polymeric stretch subsequently grows as $A_{z z}=A_{z z}^{0} \exp \left(t / 3 \lambda_{Z}\right)$. Rearranging Eq. (16) then leads to the following expression for the evolution in the midpoint diameter of the column:

$$
\frac{D_{\text {mid }}(t)}{D_{1}}=\left(\frac{A_{z z}^{0}}{A_{z z}(t)}\right)^{1 / 4} \exp \left(-\frac{t}{4 \lambda_{Z}}\right)=\exp \left(-\frac{t}{3 \lambda_{Z}}\right) .
$$

As the column necks down, and the elastic stresses in the fluid grow, the left-hand side of Eq. (17) becomes increasingly negligible and the solution smoothly crosses over to the elasto-capillary balance found by Hinch and Entov with no change in the rate of necking. Numerical simulations of the full multimode FENE-P model using nonzero initial values for the components of all modes $\mathbf{A}_{i}$ support the form of Eq. (19). The necking rate in the column rapidly adjusts so that the initial stretch in all of the higher modes relax and the capillary pressure is balanced by the viscous solvent and the longest polymeric mode.

Values of the initial stretch $A_{z z}^{0}$ computed for the three SM fluids using Eq. (18) are given in Table II. The predicted evolution in the filament diameter given by numerical simulation of Eqs. (10)-(15) for each fluid (with $N_{n}=8$ and initial conditions given by the tabulated values of $A_{z z}^{0}$ ) is shown in Fig. 6 by the solid lines. The analytic result of Eq. (19) is shown for the case of the SM-3 fluid by the dotted line. The deviation between the analytic result and numerical computations at long times is a result of the finite extensibility of the chains. It can be seen from Fig. 6 that the agreement between experi- 
ments and theoretical predictions is now nearly quantitative, with a greatly improved prediction of the time to breakup. Using linear regression to the simulation diameter data, we obtain corresponding values of the characteristic relaxation time and the time to breakup that would be measured in a capillary thinning experiment. The values obtained for $\bar{\lambda}$ and $S$ are given in Table III, and agree very well with the experimental values.

\section{THE APPARENT EXTENSIONAL VISCOSITY}

\section{A. Transient extensional stress growth}

The thinning dynamics of the elastic fluid filaments result from an interplay between the fluid rheology and the effects of capillarity. In the previous section, we have demonstrated that the observed evolution of the midfilament diameter as the fluid thread necks down and breaks is well described using a multimode FENE dumbbell model and an appropriate force balance on the fluid filament, if the initial deformation of the polymer chains is correctly accounted for. The transient extensional rheology of the fluid is encoded in this evolution and it is desirable to reexpress the midfilament diameter data in a more intuitive format, i.e., as a transient tensile stress or extensional viscosity. Following the ideas proposed by Schümmer and Tebel (1983), we can indeed obtain an apparent extensional viscosity that is related directly to the midfilament diameter by substituting Eq. (15) into the force balance given in Eq. (14). We define an appropriate apparent extensional viscosity by

$$
\bar{\eta}_{\text {app }} \equiv \frac{\Delta \tau_{p}(t)}{\dot{\varepsilon}(t)}+3 \eta_{s}
$$

Simplifying, we find that $\bar{\eta}_{\text {app }}$ is related to the first derivative of the midfilament diameter according to the following equation:

$$
\bar{\eta}_{\mathrm{app}}=\frac{2 \sigma / D_{\mathrm{mid}}(t)}{\dot{\varepsilon}(t)}=\frac{-\sigma}{d D_{\text {mid }} / d t} .
$$

To use the latter relationship, it is necessary to differentiate experimental diameter data which becomes increasingly noisy as the filament diameter decreases toward the resolution limit of the detector. In the present study, a global functional form of the midfilament diameter has been assumed that is consistent with the expected asymptotic behavior predicted by the FENE-P simulations. As we have shown, the midfilament diameter decreases exponentially at short and intermediate times, and Entov and Hinch (1997) show that the diameter will approach linear behavior at late times. This behavior results when the polymer chains become fully stretched, and the elastic stresses can no longer grow to resist the increasing capillary pressure. In this regime, the fluid behaves as a very viscous anisotropic Newtonian fluid with a viscosity equal to the steady-state extensional viscosity of the fluid $\bar{\eta}_{\infty}$. To mimic the expected diameter behavior in these two regimes, we assume the following functional form for the diameter:

$$
\frac{D_{\text {mid }}(t)}{D_{1}}=a e^{-b t}-c t+d,
$$

where $a, b, c$, and $d$ are fitting parameters. The value of $b^{-1}$ is clearly related to the longest relaxation time of the fluid, and the steady-state extensional viscosity is related to the value of $c^{-1}$. The result of fitting this functional form to the data is shown by the dashed line in the inset of Fig. 6. The apparent extensional viscosity can now be com- 


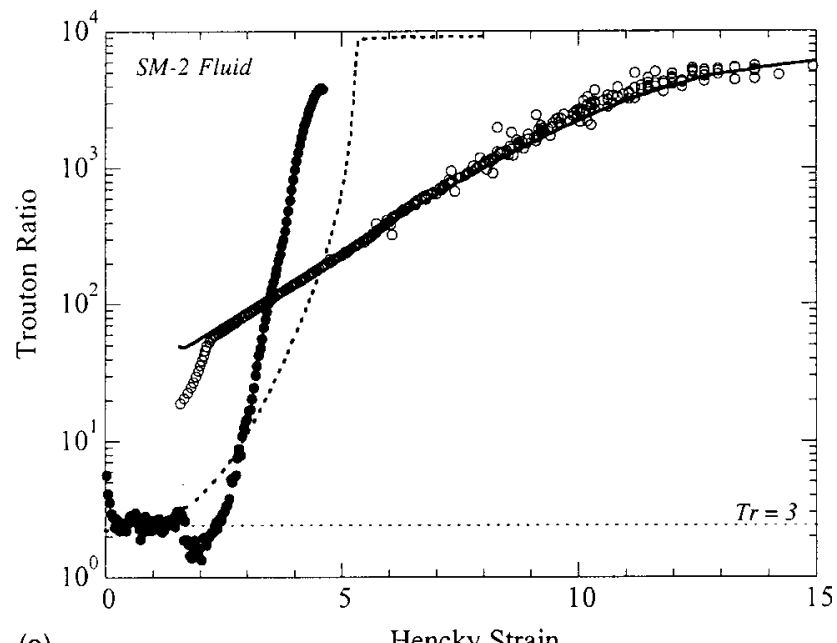

(a)

Hencky Strain

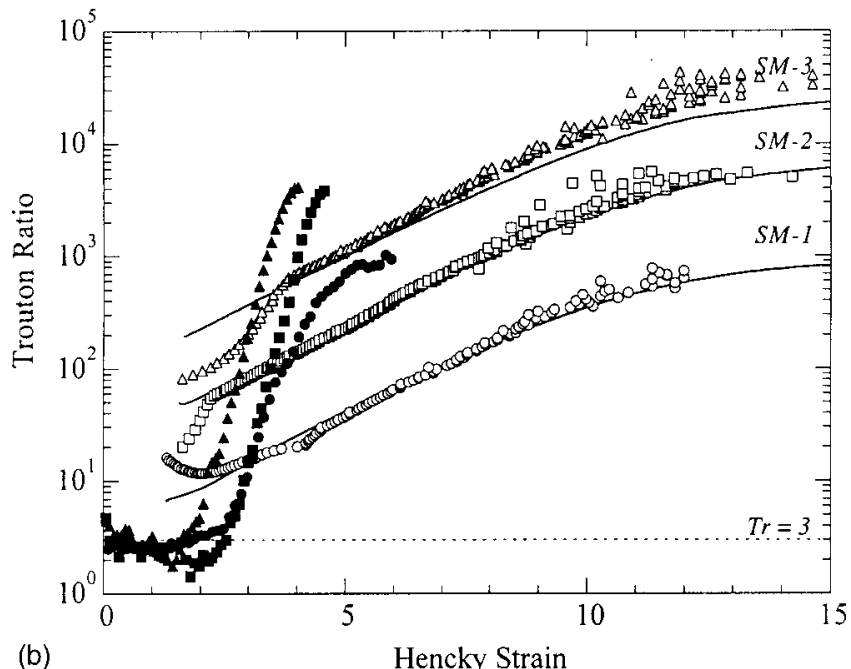

FIG. 7. Direct comparison of transient extensional viscosities measured in filament stretching rheometry with apparent extensional viscosities computed from diameter profiles of thinning filaments. (a) SM-2 Fluid. Measured extensional viscosities in filament stretching $(-\mathrm{De}=15.2)$ and filament thinning $(O, \mathrm{De} \approx 2 / 3)$ are compared with predictions from multimode FENE-P calculations. The growth of extensional viscosity is much slower during elasto-capillary thinning (solid line) due to a much smaller effective stretch rate. (b) Transient extensional viscosities for all three SM Fluids $[\mathrm{SM}-1, \mathrm{De}=17.0(\mathrm{O}), \mathrm{De} \approx 2 / 3(\bullet)$; SM-2, De $=15.2(\square)$, $\mathrm{De} \approx 2 / 3(\mathbf{\square}) ; \mathrm{SM}-3, \mathrm{De}=21.2(\triangle), \mathrm{De} \approx 2 / 3(\boldsymbol{\Delta})]$.

puted by regressing the experimental midfilament diameter to Eq. (22), differentiating, and substituting the resulting expression into Eq. (21). The diameter data at early viscous times $\left(t \leqslant \lambda_{Z}\right)$ when the initial force in the column is still important and the liquid bridge is still rearranging can be separately fit to a polynomial if desired, and the two regions connected together by requiring that the diameter and its derivative are continuous.

The transient Trouton ratio, or dimensionless extensional viscosity, for the SM-2 Boger fluid is shown in Fig. 7(a) as a function of the Hencky strain, where the Trouton ratio is defined as 


$$
\operatorname{Tr}=\frac{\bar{\eta}_{\text {app }}}{\eta_{0}}
$$

and the true Hencky strain $\varepsilon$ is given in Eq. (1). In Fig. 7(a), the hollow symbols correspond to experimental data for elasto-capillary thinning, and the solid curve corresponds to the FENE-P simulation. The initial axial step stretch imposed on the filament corresponds to a prestrain of $\varepsilon_{1}=2 \ln \left(D_{0} / D_{1}\right)$ and is indicated by the horizontal offset in the data. The experimentally measured and numerically computed midfilament diameter data were both processed using the global fitting method described above. The validity of the functional form of Eq. (22) is also verified by regressing it to the numerically computed values of $D_{\text {mid }}(t)$ and then using Eq. (21) to compute a transient extensional viscosity. Direct comparison with the stresses computed from numerical integration of Eqs. (10)-(15) yields quantitative agreement as expected.

The experimental and theoretical values for the apparent extensional viscosities extracted from elasto-capillary thinning agree well, which is consistent with the agreement observed in Fig. 6. Finally, we also show in Fig. 7(a) by filled symbols the transient Trouton ratio profiles for the SM-2 fluid experimentally measured in the filament stretching apparatus. These filament stretching data have been presented elsewhere as part of an interlaboratory comparison of filament stretching devices [Anna et al. (2000)], and are reproduced here for purposes of comparison. The experimental data terminate at a Hencky strain of $\varepsilon \approx 4$ due to onset of an elastic endplate instability that peels the elastic filament off the end fixtures of the device. The dashed curve in Fig. 7(a) shows the theoretical prediction corresponding to the filament stretching data, as predicted by the FENE-PM constitutive model [Wedgewood et al. (1991)].

Although the transient extensional viscosities derived from the filament stretching and filament thinning experiments do not appear to agree in Fig. 7(a), the two curves do approach nearly the same steady-state plateau value. The differences in the approach to steady state can be understood by recognizing that the rate of stretching in each experiment is very different. In the filament stretching experiment, the fluid filament is actively deformed at a constant (and high) rate $\dot{E}$, and for the data set presented here the dimensionless stretch rate, or Deborah number, has a value of De $\equiv \lambda_{Z} \dot{E}=15.2$. As we discuss in the companion paper, the operating range of the filament stretching device is limited not only by mechanical constraints but also by the specific rheology of the dilute solution being tested [Anna et al. (2000)]. In particular, the rate of stretching must exceed the rate of gravitational sagging such that $\mathrm{De} \geqslant \mathrm{De}_{\mathrm{sag}} \equiv \lambda_{1} \rho g D_{0} / 2 \eta_{0}$. For the SM-2 fluid with plate diameters of $D_{0}=3 \mathrm{~mm}$ this corresponds to $\mathrm{De}=10.2$.

In the filament thinning experiment, on the other hand, the kinematics are not imposed and the fluid filament is initially rapidly deformed during the step stretch and then subsequently allowed to select its own rate of necking. Although the instantaneous stretch rate in a filament thinning experiment is, in general, time varying, at short and intermediate times the deformation rate given by the analytic solution [c.f. Eq. (19)] is constant with $\lambda_{Z} \dot{\varepsilon}=2 / 3$. As Entov and Hinch (1997) explain, the deformation rate slightly exceeds the critical value for the coil-stretch transition in order for the viscoelastic stresses to exactly balance the slowly increasing capillary pressure in the thread. The apparent extensional viscosity is then found by substituting Eq. (19) into Eq. (21) to give

$$
\operatorname{Tr}_{\text {app }}=\frac{\bar{\eta}_{\text {app }}}{\eta_{0}}=3\left(\frac{\lambda_{Z} \sigma}{2 D_{1} \eta_{0}}\right) \exp \left(\frac{t}{3 \lambda_{Z}}\right) \text {. }
$$


For ideal uniaxial elongational flow, the evolution in the stretch of a Hookean dumbbell is of the general form $A_{z z} \sim A_{z z}^{0} \exp [2 \varepsilon(1-1 /(2 \mathrm{De}))]$. For filament stretching experiments at large imposed $\mathrm{De}=\lambda_{1} \dot{E} \gg 1$, the rate of increase in the extensional viscosity thus becomes $\operatorname{Tr} \sim \exp (2 \varepsilon)$. This is much faster than the rate of increase expected to be observed in elasto-capillary necking. Noting that the deformation rate is constant so that $\varepsilon \equiv \dot{\varepsilon} t=2 t /\left(3 \lambda_{Z}\right)$ we thus find from Eq. (24) that $\operatorname{Tr}_{\text {app }} \sim \exp (\varepsilon / 2)$. These estimates of the expected slopes are in excellent agreement with the measured self-thinning data. The small deviations between the data and the theoretical estimate at short times arises from gravitational sagging, axial nonuniformity, and other effects that cannot be captured in this one-dimensional theory.

In Fig. 7(b) we show the corresponding transient Trouton ratios for all three SM Boger fluids, including both filament thinning and filament stretching data. The trends in this plot are the same as those observed in Fig. 7(a). However, for the higher elasticity fluids, the filament thinning data provide a more complete measure of the transient response than can be obtained from filament stretching experiments. Gravitational sagging and elastic endplate instabilities prevent the filament stretching data from reaching a steady state plateau for the SM-2 and SM-3 fluids. Measurements of elasto-capillary thinning provide a way of extending the experimentally attainable operating space in order to obtain additional information about the extensional rheology of highly elastic fluids.

\section{B. The steady-state extensional viscosity}

At long times, finite extensibility effects become important and both experiments and theory show that the thread radius ultimately decreases linearly in time. This linear decrease at long times was also recently documented by Stelter et al. (1999). Consideration of Eq. (21) shows that although the local stretching rate in the column diverges as the breakup event is approached, the apparent extensional viscosity will approach a finite steady-state value. The global fit to the transient midfilament diameter given in Eq. (22) can be used to extract a value for this steady-state Trouton ratio for each SM Boger fluid. Although a true steady-state value can be found from the asymptotic behavior of Eq. (22) at late times, we report a "physical" steady-state value that corresponds to the maximum Hencky strain achievable by a real fluid element. From a continuum mechanics standpoint the radial extent of a fluid filament cannot realistically decrease below about $D$ $\sim 50 \mathrm{~nm}$ in size (approximately the radius of gyration of a polystyrene chain), and hence the Hencky strain cannot realistically increase above about $\varepsilon \approx 18$. This maximum Hencky strain is substituted into Eq. (22) and the corresponding maximum Trouton ratio is taken to be the steady-state Trouton ratio $\operatorname{Tr}_{\infty}$. The resulting values extracted from fits to both experiments and simulations are given in Table III.

The molecular weight dependence of the steady-state Trouton ratio is examined in Fig. 8. In order to fairly compare the molecular weight scaling of $\operatorname{Tr}_{\infty}$ with the behavior predicted from bead-spring models, the solvent contribution is removed and the result is plotted as a dimensional extensional viscosity. Brownian dynamics simulations of beadrod and bead-spring chains and asymptotic analysis of approximate closed-form models such as the FENE-PM chain show that in elongational flows the tensile stress at large strains is all carried by the longest relaxation mode [Doyle et al. (1998); Li et al. (2000)]. The steady-state extensional viscosity can thus be written in the form

$$
\bar{\eta}_{\infty}-3 \eta_{s}=2 n k T \lambda_{1} L^{2}\left(1-1 /\left(2 \lambda_{1} \dot{\varepsilon}\right)+\ldots\right)
$$




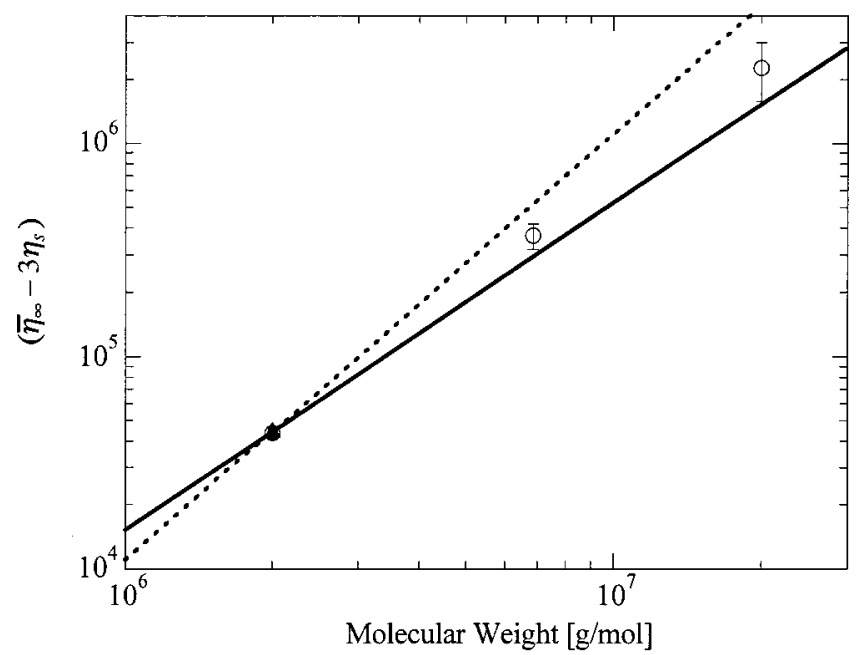

FIG. 8. Values of the steady-state extensional viscosity extracted from elasto-capillary thinning experiments $(\bigcirc)$, as a function of polymer molecular weight. The solid and dashed lines show the predictions of the Zimm and Rouse theories, respectively. Also shown is the steady-state extensional viscosity for the SM-1 fluid, extracted from a filament stretching experiment $(\boldsymbol{\Lambda})$.

where $\lambda_{1}$ is the longest relaxation time constant in the fluid. The number density $n=c N_{A} / M_{w}$, the finite extensibility $L^{2}$, and the relaxation time $\lambda_{1}$ are all functions of the molecular weight of the chain. For dilute solutions of linear monodisperse homopolymers, we expect the molecular weight dependence of the variables in Eq. (25) to be consistent with Zimm-like behavior, and the polymeric contribution to the steady-state extensional viscosity should therefore scale as $\left(\bar{\eta}_{\infty}-3 \eta_{s}\right) \sim M_{w}^{\nu+1}$ [Bird et al. (1987); Larson (1988)]. The value of the excluded volume exponent has been found from a linear regression to the viscometric data for the SM fluids to be $\nu=0.53 \pm 0.015$ [Anna et al. (2001)]. Thus, we expect the extensional viscosity to scale as $\left(\bar{\eta}_{\infty}-3 \eta_{s}\right) \sim M_{w}^{1.53}$ to be consistent with the Zimm-like scaling observed in the linear viscoelastic data. Gupta and co-workers (2000) have recently presented an extensive study of the shear and extensional rheology of a series of dilute and semidilute monodisperse polystyrene solutions. When the polymer chains are close to their equilibrium configuration, linear viscoelastic measurements were found to be well described by a Zimm spectrum of relaxation times. However, Gupta and co-workers also found that for chain conformations far from equilibrium, the molecular weight dependence of the transient and steady-state extensional viscosities measured in a filament stretching device was more consistent with a Rouse bead-spring model. In the Rouse model, the steady-state extensional viscosity given in Eq. (25) is expected to scale as $\left(\bar{\eta}_{\infty}-3 \eta_{s}\right) \sim M_{w}^{2}$.

The steady-state extensional viscosity values extracted from the filament thinning experiments are shown as hollow symbols in Fig. 8, with error bars corresponding to the noise level in the diameter sensor at late times. The solid line shown in Fig. 8 corresponds to the molecular weight dependence expected for Zimm-like scaling, while the dashed line corresponds to that of the Rouse model. A linear regression to the experimental data for all three test fluids yields the following dependence on the polymer molecular weight:

$$
\bar{\eta}_{\infty}-3 \eta_{s}=(6.4 \pm 1.1) \times 10^{-7} M_{w}^{(1.720 \pm 0.010)} .
$$


The fitted scaling exponent lies inbetween the values expected for the Rouse and Zimm models, suggesting that as the polymer molecules approach near full extension, the hydrodynamic interaction of the polymer chains with the surrounding solvent may be reduced [Gupta et al. (2000)]. Furthermore it is clear from Fig. 8 that the deviation from Zimm scaling toward Rouse scaling becomes systematically more pronounced at very high molecular weight. Li et al. (2000) discuss further possible mechanisms for this effect at very high molecular weights. Finally, the single solid symbol in Fig. 8 corresponds to the steady-state extensional viscosity of the SM-1 fluid extracted from filament stretching experiments [Anna et al. (2000)]. This value agrees quantitatively with the corresponding filament breakup value.

\section{CONCLUSIONS}

In the present study, we have examined the dynamics of elasto-capillary thinning for three different model elastic liquids. The evolution in the midpoint diameter profile of necking and breaking fluid filaments was measured in a filament stretching device by elongating an initially cylindrical fluid filament to a predetermined Hencky strain and then monitoring the evolution in $D_{\text {mid }}(t)$ using a laser micrometer. After an initial transient relaxation of the viscoelastic stress associated with stretching the filament, the midfilament diameter profiles of all three Boger fluid filaments are observed to decay exponentially in time, in contrast to the linear evolution in the diameter of a Newtonian fluid filament. For elastic fluids with a spectrum of relaxation times that has been well characterized in linear viscoelastic measurements, we find that the relaxation time extracted from measurements of elasto-capillary thinning agrees quantitatively with the longest (Zimm) time constant.

Recently, Kolte and Szabo (1999) have shown that incorporating radial gradients in the axial stress of the form $\tau_{z z} \sim \kappa_{1}+\kappa_{2} r^{\beta}$ (where $\kappa_{1}$ and $\kappa_{2}$ are constants) leads to a reduction in the rate of necking such that $D_{\text {mid }}(t) \sim \exp \left[-t /\left((3+\beta) \lambda_{Z}\right)\right]$. Conversely our experiments indicate that the longest time constant $\bar{\lambda}$ extracted from diameter data during the intermediate elastic regime is in fact slightly less than the value of $\lambda_{Z}$ extracted from linear viscoelastic data. This would appear, from the analysis of Kolte and Szabo at least, to indicate that $\beta<0$; however, a more likely explanation is that the evolution in the diameter profile at intermediate times is still weakly affected by the shorter time constants $\lambda_{i}=\lambda_{Z} / i^{3 \nu}$ of the Zimm spectrum. Inspection of Eq. (8) shows these will always serve to increase the slope of $D_{\text {mid }}(t)$, and thus reduce the fitted value of $\bar{\lambda}$. The theory of Entov and Hinch (1997) (appropriately modified to account for nonzero initial stretches) and our experiments both show that as the molecular weight increases, the dimensionless time to breakup $S$ also increases. The extent of the "intermediate" elastic regime increases concomitantly and the value of $\bar{\lambda}$ should approach $\lambda_{Z}$ ever more closely. Indeed the data in Table III show that the relative error $\left(\lambda_{Z}-\bar{\lambda}\right) / \lambda_{Z}$ decreases from $14 \%$ for SM-1 to $2 \%$ for SM-3. Numerical simulations exploring the effect of aspect ratio show that radial stress gradients become increasingly severe as the initial aspect ratio $\Lambda_{0}$ decreases [Yao and McKinley (1998)]. The initial aspect ratio used in the present experiments was $\Lambda_{0}=1$, whereas Kolte and Szabo used a smaller value of $\Lambda_{0}=0.71$.

The experimental diameter profiles are also compared with predictions computed using a multimode FENE-P dumbbell model and a force balance on the fluid filament that includes viscous, elastic, and capillary forces. Only qualitative agreement is obtained with the predicted evolution in the filament diameter if the initial stresses are assumed to 
be zero; however, by using a nonzero estimate of the initial stresses, the evolution in the midfilament diameters can be predicted almost quantitatively. This estimate of the initial tensile stress difference can be based either on the tensile force measured experimentally at the instant that stretching stops (if it is available), or by using an analytic approximation [c.f. Eq. (18)] for the initial deformation of the polymer chains.

Finally, we show how the force balance on the fluid filament can be rearranged to compute an apparent extensional viscosity from the measured midfilament diameter profile. The resulting curves for the transient extensional viscosity approach the same steadystate plateau as corresponding curves from filament stretching experiments. The transient growth of the extensional viscosities for the filament thinning and filament stretching experiments is consistent with asymptotic predictions corresponding to low and high deformation rates, respectively. However, the dependence of the steady-state extensional viscosity on the molecular weight of the polymeric solute is not quite in agreement with the scaling of the bead-spring constitutive equation when the Zimm model is used to describe the variation in the relaxation time and the polymeric contribution to the shear viscosity. Instead, the molecular weight dependence lies inbetween the limiting curves corresponding to Zimm-like behavior and Rouse-like behavior, suggesting that hydrodynamic interaction is important for small deformations of the polymer molecules, but that free draining behavior may be enhanced as the polymer molecules become fully stretched. For fluids with more complex topologies than the simple monodisperse homopolymer solutions considered in the present work, the steady-state Trouton ratio will also depend on additional structural parameters such as the local backbone rigidity, the degree of branching, or grafting density [see, for example, Stelter et al. (1999)].

Filament stretching and filament thinning experiments yield consistent and complementary extensional rheological information about a viscoelastic fluid. The filament stretching experiment imposes an extensional flow with a well-defined time scale $\dot{E}^{-1}$, whereas in elasto-capillary thinning the necking filament selects a natural scale $\dot{E}$ $\sim 2 /\left(3 \lambda_{1}\right)$. As a result, filament stretching data are somewhat easier to interpret; but, in practice, motor- and fluid-related constraints limit the useful operating space of the device. The filament breakup experiment, on the other hand, is relatively simple to perform, but more complicated to interpret. Through a detailed study of the behavior of model fluids in each device, we have shown that elasto-capillary thinning experiments can help extend the operating space of a filament stretching experiment and can yield useful quantitative information about the extensional rheology of a fluid. It is not possible to use the dilute solution theory discussed above to interpret necking measurements for other viscoelastic test fluids such as concentrated solutions and melts without selecting unreasonably low values of parameters such as the dumbbell extensibility L. However, it is always possible to use Eqs. (18) and (19) to compute an apparent extensional viscosity for the material being tested.

This combined approach of using data obtained from both filament devices should also prove useful in understanding the extensional behavior of other viscoelastic fluids. For example, transient extensional viscosity curves obtained in a filament stretching experiment are analogous to "tack" curves commonly used to characterize the behavior of adhesives [Crosby and Shull (1999)]. The fibrils that subsequently form during peeling and failure of adhesives [Gay and Leibler (1999) are also similar to those observed in the filament stretching rheometer at high strains and strain rates [Spiegelberg and McKinley (1996); Ferguson et al. (1997)]. Thus, filament stretching and thinning experiments are expected to be useful tests to help quantify the role of extensional rheology in adhesion processes. We believe that a combined approach utilizing data from both devices will 
provide useful insight both for academic researchers interested in the extensional response of model viscoelastic liquids, as well as for industrial researchers who need a simple, yet quantitative probe of the extensional rheology of viscoelastic materials.

\section{ACKNOWLEDGMENTS}

This research was supported by NASA under Grant Nos. NAG3-1385 and NCC3-610. S. L. A. thanks the Fannie and John Hertz Foundation for supporting her graduate research. The authors would like to thank Professor S. J. Muller for preparing the test fluids used in this study, Professor V. Entov for helpful and spirited discussions, and the anonymous referees for their insightful comments.

\section{References}

Anna, S. L., C. Rogers, and G. H. McKinley, "On controlling the kinematics of a filament stretching rheometer using a real-time active control mechanism,'” J. Non-Newtonian Fluid Mech. 87, 307-335 (1999).

Anna, S. L., G. H. McKinley, D. A. Nguyen, T. Sridhar, S. J. Muller, J. Huang, and D. F. James, "An inter-laboratory comparison of measurements from filament-stretching rheometers using common test fluids,"' J. Rheol. 45, 83-114 (2001).

Bazilevsky, A. V., V. M. Entov, and A. N. Rozhkov, "Liquid filament microrheometer and some of its applications," in Third European Rheology Conference, edited by D R. Oliver (Elsevier, New York, 1990), pp. 41-43.

Bazilevsky, A. V., V. M. Entov, M. M. Lerner, and A. N. Rozhkov, "Failure of polymer solution filaments,' Polym. Sci., Ser. A Ser. B 39, 316-324 (1997).

Berg, S., R. Kröger, and H. J. Rath, "Measurement of extensional viscosity by stretching large liquid bridges in microgravity," J. Non-Newtonian Fluid Mech. 55, 307-319 (1994).

Bird, R. B., C. F. Curtiss, R. C. Armstrong and O. Hassager, Dynamics of Polymeric Liquids, Volume 2: Kinetic Theory, 2nd ed. (Wiley, New York, 1987).

Bogy, D. B., “Drop formation in a circular liquid jet,” Annu. Rev. Fluid Mech. 11, 207-228 (1979).

Bousfield, D. W., R. Keunings, G. Marrucci, and M. M. Denn, "Nonlinear analysis of the surface-tension driven breakup of viscoelastic fluid filaments," J. Non-Newtonian Fluid Mech. 21, 79-97 (1986).

Brenner, M. P., J. R. Lister, and H. A. Stone, "Pinching threads, singularities and the number $0.0304 \ldots$..., Phys. Fluids 8, 2827-2836 (1996).

Chang, H. and A. S. Lodge, "Comparison of rubber-like liquid theory with stress-growth data for elongation of a low-density branched polyethylene melt," Rheol. Acta 11, 127-129 (1972).

Chang, H-C., E. A. Demekhin, and E. Kalaidin, "Iterated stretching of viscoelastic jets," Phys. Fluids 11, 1717-1737 (1999).

Crosby, A. J. and K. R. Shull, "Adhesive failure analysis of pressure-sensitive adhesives," J. Appl. Polym. Sci. 37, 3455-3472 (1999).

Doyle, P., E. S. G. Shaqfeh, G. H. McKinley, and S. H. Spiegelberg, "Relaxation of dilute polymer solutions following extensional flow," J. Non-Newtonian Fluid Mech. 76, 79-110 (1998).

Eggers, J., 'Nonlinear dynamics and breakup of free-surface flows,'” Rev. Mod. Phys. 69, 865-929 (1997).

Entov, V. M. and E. J. Hinch, "Effect of a spectrum of relaxation times on the capillary thinning of a filament of elastic liquid,” J. Non-Newtonian Fluid Mech. 72, 31-54 (1997).

Ferguson, J., B. Reilly, and N. Granville, "Extensional and adhesion characteristics of a pressure sensitive adhesive," Polymer 38, 795-800 (1997).

Gay, C. and L. Leibler, “'On stickiness,” Phys. Today 52, 48-52 (1999).

Goldin, M., H. Yerushalmi, R. Pfeffer, and R. Shinnar, "Breakup of a laminar capillary jet of a viscoelastic fluid,', J. Fluid Mech. 38, 689-711 (1969).

Goren, S. L. and M. Gottlieb, "Surface-tension-driven breakup of viscoelastic liquid threads," J. Fluid Mech. 120, 245-266 (1982).

Gupta, R. K., D. A. Nguyen, and T. Sridhar, "Extensional viscosity of dilute polystyrene solutions—effect of concentration and molecular weight," Phys. Fluids 12, 1296-1318 (2000).

Ide, Y. and J. L. White, "The spinnability of polymer fluid filaments," J. Appl. Polym. Sci. 20, 2511-2531 (1976).

Kolte, M. I. and P. Szabo, "Capillary thinning of polymeric filaments," J. Rheol. 43, 609-625 (1999).

Kolte, M. I., H. K. Rasmussen, and O. Hassager, "Transient filament stretching rheometer II: Numerical simulation," Rheol. Acta 36, 285-302 (1997). 
Kröger, R., S. Berg, A. Delgado, and H. J. Rath, "'Stretching behavior of large polymeric and Newtonian liquid bridges in plateau simulation," J. Non-Newtonian Fluid Mech. 45, 385-400 (1992).

Larson, R. G., Constitutive Equations for Polymer Melts and Solutions (Butterworths, Boston, 1988).

Li, L., R. G. Larson, and T. Sridhar, "Brownian dynamics simulation in dilute polystyrene solutions,'” J. Rheol. 44, 291-322 (2000).

Liang, R. F. and M. R. Mackley, "Rheological characterization of the time and strain dependence for polyisobutylene solutions," J. Non-Newtonian Fluid Mech. 52, 387-405 (1994).

Matta, J. E. and R. P. Tytus, "Liquid stretching using a falling cylinder,"' J. Non-Newtonian Fluid Mech. 35, 215-229 (1990).

McKinley, G. H. and A. Tripathi, "How to extract the Newtonian viscosity from capillary breakup measurements in a filament rheometer," J. Rheol. 44, 653-670 (2000).

Middleman, S., "Stability of a viscoelastic jet," Chem. Eng. Sci. 20, 1037-1040 (1965).

Orr, N. V. and T. Sridhar, "Probing the dynamics of polymer solutions in extensional flow using step strain rate experiments,' J. Non-Newtonian Fluid Mech. 82, 203-232 (1999).

Papageorgiou, D. T., '"On the breakup of viscous liquid threads,"' Phys. Fluids 7, 1529-1544 (1995).

Rayleigh, L., (J. W. Strutt), “On the instability of jets," Proc. London Math. Soc. 10, 4-13 (1878).

Rayleigh, L., (J. W. Strutt), “'On the stability of a cylinder of viscous liquid under capillary force,' Philos. Mag. 34, 145 (1892).

Renardy, M., "Some comments on the surface-tension driven breakup (or the lack of it) of viscoelastic jets," J. Non-Newtonian Fluid Mech. 51, 97-107 (1994).

Renardy, M., "A numerical study of the asymptotic evolution and breakup of newtonian and viscoelastic jets," J. Non-Newtonian Fluid Mech. 59, 267-282 (1995).

Schümmer, P. and K. H. Tebel, "A new elongational rheometer for polymer solutions," J. Non-Newtonian Fluid Mech. 12, 331-347 (1983).

Solomon, M. J. and S. J. Muller, "The transient extensional behavior of polystyrene-based Boger fluids of varying solvent quality and molecular weight,"' J. Rheol. 40, 837-856 (1996).

Spiegelberg, S. H. and G. H. McKinley, "Stress relaxation and elastic decohesion of viscoelastic polymer solutions in extensional flow," J. Non-Newtonian Fluid Mech. 67, 49-76 (1996).

Spiegelberg, S. H., D. C. Ables, and G. H. McKinley, "The role of end-effects on measurements of extensional viscosity in viscoelastic polymer solutions with a filament stretching rheometer," J. Non-Newtonian Fluid Mech. 64, 229-267 (1996).

Sridhar, T., V. Tirtaatmadja, D. A. Nguyen, and R. K. Gupta, "Measurement of extensional viscosity of polymer solutions," J. Non-Newtonian Fluid Mech. 40, 271-280 (1991).

Stelter, M., T. Wunderlich, S. K. Rath, G. Brenn, A. L. Yarin, R. P. Singh, and F. Durst, “'Shear and extensional investigations of solutions of grafted/ungrafted amylopectin and polyacrylamide,' J. Appl. Polym. Sci. 74, 2773-2782 (1999).

Stelter, M., G. Brenn, A. L. Yarin, R. B. Singh, and F. Durst, "Validation and application of a novel elongation device for polymer solutions," J. Rheol. 44, 595-616 (2000).

Szabo, P., "Transient filament stretching rheometer I: Force balance analysis," Rheol. Acta 36, 277-284 (1997).

Tirtaatmadja, V. and T. Sridhar, "A Filament stretching device for measurement of extensional viscosity," J. Rheol. 37, 1081-1102 (1993).

Tomotika, S., "On the instability of a cylindrical thread of a viscous liquid surrounded by another viscous fluid," Proc. R. Soc. London, Ser. A 150, 322-337 (1935).

van Nieuwkoop, J. and M. M. O. Muller von Czernicki, "Elongation and subsequent relaxation measurements on dilute polyisobutylene solutions," J. Non-Newtonian Fluid Mech. 67, 105-124 (1996).

Verhoef, M. R. J., B. H. A. A. van den Brule, and M. A. Hulsen, "On the modeling of a PIB/PB Boger fluid in extensional flow," J. Non-Newtonian Fluid Mech. 80, 155-182 (1999).

Wedgewood, L. E., D. N. Ostrov, and R. B. Bird, "A finitely extensible bead-spring chain model for dilute polymer solutions," J. Non-Newtonian Fluid Mech. 40, 119-139 (1991).

Yao, M. and G. H. McKinley, "Numerical simulation of extensional deformations of viscoelastic liquid bridges in filament stretching devices," J. Non-Newtonian Fluid Mech. 74, 47-88 (1998).

Yarin, A. L., Free Liquid Jets and Films: Hydrodynamics and Rheology (Longman-Wiley, New York, 1993). 